\title{
Entramado de violencias, efectos y alternativas de docentes y estudiantes de secundaria ${ }^{3}$
}

\author{
Violence, Effects and Alternatives of Teachers and Secondary Students, a Structural Framework \\ Malha de violências, efeitos e alternativas de docentes e estudantes de secundária
}

\section{Resumen}

Con la participación de docentes de una secundaria en Morelos (México) investigamos las violencias desde los diversos ámbitos de la cotidianidad de sus actores: la colonia, la familia, la escuela y el aula, así como sus efectos en el entramado de relaciones sociales involucradas. De modo que, en este artículo abordamos las formas de violencia que los sujetos viven o expresan en el contexto escolar y algunos de sus efectos sociales y educativos. En un segundo momento, presentamos los primeros resultados de la intervención realizada en forma de 'acompañamiento entre pares' orientada a bajar los niveles de deserción y de rezago escolar. Para finalizar, proponemos las escuelas basadas en "redes de cooperación social", como un modo de enfrentar hechos estructurales y coyunturales que se sobreponen a la escuela y desde donde se construya una convivencia basada en la protección de la vida.

Palabras clave: entramado, violencias, docentes, estudiantes, cooperación.

\section{Recibido: 3 de octubre, evaluado: 4 de noviembre, aprobado: 10 de noviembre}

1 Doctora en Educación. Maestra en Psicología Clínica Infantil. Adscrita a la Universidad Pedagógica Nacional (UPN)-Morelos. Correo electrónico: gpoujol@yahoo.com.mx

2 Doctora en Ciencias Sociales. Adscrita a la Universidad Pedagógica Nacional (UPN)-Morelos. Coordinadora del Doctorado en Investigación e Intervención Educativa. Correo electrónico: myrfracchia@gmail.com

3 Artí́culo de investigación, derivado de un proceso investigación-intervención desarrollado por las autoras en el curso de este año 2016, involucrando a los docentes de la Escuela Secundaria Pública No. 5 en el Estado de Morelos (México), para favorecer la convivencia escolar. 


\begin{abstract}
With the participation of high school teachers in Morelos (Mexico), we researched violences from the different realms of its actors' everyday lives: the neighborhood, family, school and the classroom, as well as their effects in the structures of corresponding social relations. Therefore, in this article, we explore the different manifestations of violence that individuals face or express in the school context, as well as some of their social and educational effects. Additionally, we present early results from an intervention performed in the way of 'peer accompaniment' designed to lower rates of educational desertion and lagging. Finally, we propose schools based on 'social cooperation networks' as a way to deal with structural and contextual factors that superimpose themselves on schools, as well as a way to build conviviality based on the protection of life.
\end{abstract}

Keywords: Structural Framework, Violence, Teachers, Students, Cooperation.

\title{
Resumo
}

Com a participação de docentes de uma secundária em Morelos (México) pesquisamos as violências desde os diversos âmbitos da cotidianidad de seus atores: a colônia, a família, a escola e o sala, bem como seus efeitos na malha de relações sociais envolvida. De maneira que, neste artigo abordamos as formas de violência que os sujeitos vivem ou expressam no contexto escolar e alguns de seus efeitos sociais e educativos. Em um segundo momento, apresentamos os primeiros resultados da intervenção realizada em forma de "acompanhamento entre pares" orientada a baixar os níveis de deserção e de atraso escolar. Para finalizar, propomos as escolas baseadas em "redes de cooperação social", como um modo de enfrentar fatos estruturais e conjunturais que se sobrepõem à escola e desde onde se construa uma convivência baseada na proteção da vida.

Palavras-chave: malha, violências, docentes, estudantes, cooperação. 


\section{Introducción}

En este artículo presentaremos los avances más importantes de un proceso de investigación-intervención, desde el que se indaga acerca de las formas de violencia que se viven o se expresan en una escuela secundaria pública en el Estado de Morelos, México (Esc. Sec. No. 5) y algunos de los efectos sociales y educativos que tienen en docentes y estudiantes. Asimismo, parte de los resultados de la intervención realizada y, finalmente, presentamos una propuesta a estudiar que permita construir un mejor ambiente socioeducativo.

Este estudio puede aportar a las investigaciones para la paz, elementos para comprender la manera en la que se deterioran los ambientes sociales en una institución como la escuela, la red de relaciones que se establece entre los ambientes escolares y sociales, sus efectos en los sujetos, al tiempo que se identifican las estrategias y recursos con los que cuentan los actores para transformar las relaciones sociales en las que están inmersos para favorecer un clima escolar de cooperación. Como afirman (Fracchia, García, \& Cortés, 2013):

No debe asombrar la preocupación acerca del problema de las violencias expresadas en las escuelas dadas las diversas formas en que la guerra se está expresando en todo el territorio nacional, involucrando tanto al crimen organizado como al Estado para asegurarse el monopolio del narcotráfico. Es la sociedad mexicana la principal víctima de esta situación que ha acumulado ya un alarmante costo humano (Equipo Bourbaki, 2011). Esto coloca a México como uno de los territorios más violentos y vulnerables del orbe, aunada a la agudización de la crisis económica, al mayor volumen de trabajo informal respecto al trabajo formal y al aumento exponencial del desempleo. El quiebre al interior del aparato del Estado, que detenta el monopolio de las violencias física y simbólica, se refleja en todos los ámbitos, incluyendo el educativo. (pp. 1-2).

Según el Consejo Ciudadano para la Seguridad Pública y Justicia Penal, A.C., en 2014 el estado de Morelos era el más violento del país y Cuernavaca el primer municipio más violento.

\section{Antecedentes del estudio}

Este estudio tiene como antecedente la demanda de maestros de Educación Básica y Media Superior que estudian un posgrado en la Universidad Pedagógica Nacional (Morelos, México) - a la que estamos adscritas- por cómo atender las problemáticas de violencia escolar que enfrentan. Es por ello que hemos desarrollado la línea de generación y aplicación de conocimiento sobre Violencia escolar y pedagogía de la cooperación. De allí surgió la necesidad de diseñar y realizar un diplomado dirigido a docentes de una escuela secundaria a través de un proceso de investigación e intervención educativa sobre las formas de violencia y sus efectos en sus estudiantes y sobre ellos mismos.

Los avances de esta investigación exploratoria se han generado a partir del Diplomado sobre Violencias en la escuela y pedagogía de la cooperación para la ciudadanía en 2016 en una escuela secundaria en Cuernavaca, Morelos. En éste hemos realizado un diagnóstico preliminar con el conjunto de los docentes acerca de las formas de convivencia en el espacio escolar y sus efectos sociales, con el supuesto de que las relaciones sociales existentes en el espacio escolar son parte de un entramado social más amplio que involucra a las de sus participantes, lo cual tiene efectos en los espacios de su cotidianidad (Elias, 1990).

El estado del arte desarrollado por Krauskopf (2006) identifica tres formas en las que los programas de prevención de la violencia en ámbitos escolares han evolucionado a lo largo de la historia: represión y control, prevención, promoción del desarrollo humano y recuperación del capital social. Podemos ubicar este estudio como de recuperación del capital social, pues se promovió la participación, la colectividad y el protagonismo de docentes y estudiantes y otros actores de la comunidad escolar. Otros elementos que analiza esta autora se refieren a que la violencia no puede ser comprendida ni tratada de manera reducida en el ámbito escolar, es necesario considerar todos los elementos implicados. Krauskopf (2006) plantea 
que en América Latina la mayoría de los proyectos de prevención de la violencia surgen de la preocupación por la violencia de la sociedad, es por ello que las estrategias incluyen la protección de la vida y la cooperación, en este estudio compartimos esta visión.

Un rasgo dominante del entramado social es la relación entre desigualdad social, exclusión escolar y violencia. Ésta ha sido estudiada desde diferentes perspectivas, siendo una de ellas la investigación para la paz. En este campo encontramos la realizada por Francisco Jiménez (2012) quien plantea que las semillas de violencia se siembran por las condiciones sociales y los aspectos inhumanos del entorno.

Hablando de la inhumanidad, Jiménez (2012, p. 18) recurre a Galtung, quien ha interpretado la violencia como el no desarrollo de las posibilidades potenciales (somáticas y mentales) de los seres humanos. La violencia tiene un carácter multifacético y está presente en varias escalas y ámbitos, refiere Jiménez que en la economía se relaciona con la falta de recursos, explotación, discriminaciones, marginación. En la ideología, con la subordinación de la información, la manipulación de la opinión pública, la propaganda con trasfondo violento y discriminador; en la enseñanza, con autoritarismos, castigos e intransigencias.

Jiménez (2012) identifica puntos de contacto entre la violencia estructural y la violencia simbólica. En ese terreno reconoce los aportes de Marx y de Galtung en el concepto de violencia estructural que permite develar formas ocultas y estáticas de la violencia y las relaciones entre unas y otras. En este tipo de violencia no hay un sujeto agresor fácilmente identificable, pues, está enmascarado en una trama de estructuras injustas; quien la sufre no la percibe como tal, porque existen mediaciones de la violencia cultural y/o simbólica que le impiden reconocerla. Un ejemplo que da este autor acerca de cómo se relacionan la violencia estructural y la violencia simbólica es la violencia cultural referida a la escuela y a sus contenidos; en ella se impone una definición del mundo, de formas de apreciación de la realidad, apareciendo como natural y legítimo, disimulando así las relaciones de fuerza. "La ideología que se trasmite es la del hombre, blanco, urbano, de clase media o media alta integrado en la sociedad" (Jiménez, 2012, p. 42).

Vinculando la violencia estructural y simbólica, otros investigadores referidos en Poujol (2016) han identificado la relación entre la exclusión social de los jóvenes, con una gama de implicaciones personales y sociales; esta situación aunada a experiencias de discriminación, a la distancia cultural entre la vida de los jóvenes y la escuela, sus contenidos y formas de trabajo cotidiano, la arbitrariedad de las normas, la ruptura entre el mundo juvenil y la vida escolar, la normalización del maltrato y la violencia, el mínimo espacio que se brinda a la afectividad y a la participación de los estudiantes.

Jiménez (2012) sostiene que la violencia cultural es la base que sustenta la violencia directa y estructural, ya que las manifestaciones culturales son representaciones que mantienen la jerarquización social y organizan el pensamiento social. Ahondando en la perspectiva socioeducativa, Di Napoli (2009) vincula la producción simbólica de comportamientos sociales violentos en el espacio escolar a las condiciones y posibilidades de la socialización y la biografización de los estudiantes, pues, desde esta visión la violencia forma subjetividades.

Para Jiménez (2012), tomar conciencia de que la violencia simbólica asegura la dominación y justifica la violencia estructural y directa es un poder-saber que puede favorecer la producción de cambios sociales a favor de la convivencia pacífica. Si bien esas formas de violencia se pueden reconocer en la escuela, éstas no son un correlato mecánico de la violencia social, aunque es allí donde se originan y cobran sentido. Kaplan (2009) señala que en la escuela pueden hacerse ciertas mediaciones para posibilitar algo distinto a los comportamientos brutales de las sociedades capitalistas salvajes.

Compartimos con Jiménez y Kaplan la idea de que existe una relación compleja entre la violencia social y la violencia escolar, pero también la escuela puede ser un espacio alternativo para que las niñas, 
niños, jóvenes, docentes y otros miembros de la comunidad educativa, puedan construir otras formas de relación más cooperativas e incluyentes.

Es desde esta perspectiva que hemos desarrollado un proyecto de investigación e intervención, en la que han participado la mayoría de los docentes, alumnos y otros actores en una escuela secundaria del Estado de Morelos, México.

\section{Aproximación metodológica}

Se eligió la escuela secundaria por estar ubicada en uno de los polígonos de mayor índice de inseguridad en Morelos. Son frecuentes la riñas entre pandillas en esa zona. En años anteriores esa rivalidad también se manifestaba dentro de la escuela y la institución acudió a la policía para garantizar la seguridad de los estudiantes y docentes a la hora de salida.

Nuestra aproximación metodológica fue mixta, trabajamos con datos cuantitativos y cualitativos buscando una mejor comprensión del fenómeno estudiado y un acercamiento a su complejidad. El procedimiento seguido tiene como antecedente lo propuesto por Onwuegbuzie y Leech (2006), quienes plantearon que las investigaciones con un diseño mixto podían ser de dos tipos: con modelo mixto, en el cual se combinan en una misma etapa o fase de investigación, métodos cuantitativos y cualitativos, que es el que seguimos; o con un método mixto, en cuyo caso, los métodos cuantitativos se utilizan en una etapa o fase de la investigación y los cualitativos en otra.

En cuanto al método cuantitativo diseñamos dos cuestionarios, uno dirigido a 429 estudiantes de los tres grados en los turnos matutino y vespertino y otro a 51 docentes, que exploran hechos de violencia en diversos ámbitos sociales y escolares, así como los efectos de éstos en el entrevistado y su juicio moral, que aún está en procesamiento. Los datos fueron vaciados y analizados desde el programa estadístico Statistical Package for the Social Sciences (spss).

El método cualitativo se basó en registros obtenidos a lo largo del diplomado a través de los ejercicios aplicados a los 35 docentes participantes en el diplomado y a los más de 50 docentes en dos consejos técnicos; además de sus reflexiones sobre lecturas vinculantes a esta problemática; a los ejercicios que ellos han aplicado a sus estudiantes durante el proceso de 'acompañamiento de pares' y sus reflexiones sobre los mismos. Los datos obtenidos se orientaron a explorar las características de la convivencia y el clima escolar y las experiencias de docentes y alumnos tanto en el momento del diagnóstico como de la intervención educativa.

\section{Los procesos de diagnóstico e intervención educativa en la escuela secundaria}

Los cuestionarios aplicados acerca de los hechos de violencia que los docentes y sus estudiantes identificaban en su colonia, en su familia (sólo en el caso de los estudiantes), en la escuela y en su aula y acerca de los efectos que tuvieron en cada uno de estos ámbitos, ha permitido conocer el entramado de la violencia en la convivencia cotidiana desde la percepción de sus involucrados.

El interrogante acerca de si los estudiantes y docentes de la escuela objeto de estudio reconocían hechos de violencia en sus ámbitos cotidianos surgió ante el supuesto de la 'normalización de la violencia' que permea en los directivos y docentes del plantel, a raíz de lo cual no esperaban que los estudiantes reconocieran tales hechos de violencia porque, en su opinión, ya no los considerarían como tales. Un primer resultado de este estudio, en cambio, fue que para la mayoría de los encuestados sí existen hechos de violencia. Incluso los identifican con claridad para cada uno de los ámbitos considerados: en la colonia, en la familia (sólo se preguntó a los estudiantes), en la escuela y en el aula (véase tabla 1). 
Tabla 1. Estudiantes y docentes que reconocen hechos de violencia según los ámbitos

\begin{tabular}{|c|c|c|c|c|}
\hline $\begin{array}{c}\text { Hay } \\
\text { violencias }\end{array}$ & $\begin{array}{c}\text { En la } \\
\text { escuela }\end{array}$ & $\begin{array}{c}\text { En la } \\
\text { colonia }\end{array}$ & En el aula & $\begin{array}{c}\text { En la } \\
\text { familia }\end{array}$ \\
\hline Estudiantes & $\begin{array}{c}86 \% \\
(368)\end{array}$ & $\begin{array}{c}76 \% \\
(324)\end{array}$ & $\begin{array}{c}71 \% \\
(304)\end{array}$ & $\begin{array}{c}45 \% \\
(184)\end{array}$ \\
\hline Docentes & $\begin{array}{c}100 \% \\
(51)\end{array}$ & $\begin{array}{c}86 \% \\
(44)\end{array}$ & $\begin{array}{c}59 \% \\
(30)\end{array}$ & \\
\hline
\end{tabular}

Fuente: elaboración propia (2016).

La gran mayoría de docentes y estudiantes señala como el lugar donde se expresa el mayor grado de violencia a la escuela, luego a la colonia, al aula y, finalmente, a su familia (sólo los estudiantes). Lo que varía entre estudiantes y docentes es la intensidad de la respuesta: la existencia de hechos violentos en la escuela es afirmado por casi nueve de cada 10 estudiantes y por la totalidad de los docentes. En la colonia, sin embargo, son los docentes más que los estudiantes, los que señalan esos hechos; mientras que en el aula (aún si para ambos sujetos es un espacio de menor violencia que en el resto de la escuela) son más los estudiantes que los docentes en señalarlo: siete de cada diez estudiantes contra casi seis de cada diez docentes.

Desde aquí empezamos a mostrar que los sujetos tienen claridad al nombrar los hechos de violencia, que la normalización de la violencia existente no ha llegado al punto de encubrirlos y que localizan estos hechos y los diferencia por ámbito de convivencia cotidiana.

Respecto a los efectos que estos hechos señalados como violentos tienen sobre ellos, ciertamente, han sido más los estudiantes y docentes que consideran que no han sido afectados por éstos, en cuanto ser afectados es considerado sólo cuando alguno de ellos ha sido objeto de estos actos violentos de manera directa o alguno de los miembros de su familia, amigos o conocidos.

En este artículo nos referiremos a los hechos violentos mencionados por el conjunto de docentes y estudiantes encuestados y a los efectos que de manera directa se ha ejercido sobre una parte de ellos.

\section{Hechos de violencia por ámbito, reconocidos por los estudiantes y docentes, y formas en que les afecta $^{4}$}

\section{En la colonia}

Para los docentes encuestados, el hecho de violencia más nombrado ha sido el robo, sobre todo el de la casa habitación; mencionan, además, los asesinatos, secuestros, peleas y el narcomenudeo. El robo le ha afectado a casi la mitad de ellos, de manera directa, y los demás hechos de manera indirecta. Su principal efecto ha sido la siembra del miedo y el terror que les produce salir y regresar a la colonia y a su casa; asimismo, la falta de tranquilidad, la pérdida de confianza en la gente, la vida incierta ya que "todos estamos expuestos". También los estudiantes mencionan el conjunto de estos hechos en su colonia, pero agregan otros más, los de violación, maltrato, levantón y desaparición. Casi un tercio de los estudiantes afirman que estos hechos les han afectado, de manera directa o indirecta. Mencionan, además, peleas y golpes, robos y asaltos; asesinatos; violaciones o intento de violación; 'levantones' (o secuestros en donde no se pide rescate, sino que se desaparece a la persona) y secuestros; maltrato y 'problemas de violencia', que no especifican y, finalmente, la drogadicción en las calles.

Los estudiantes afectados por hechos de violencia en la colonia tienen miedo frente a lo que les puede suceder directamente en sus propios cuerpos o en el de sus familiares. Tienen miedo:

- A ser asesinados: "tengo que estar cuidándome para que no me maten, es que mataron a un tío". Otro presenció un asesinado, las balaceras afectaron su casa.

4 Todos los relatos que aparecen en este apartado, provienen de las respuestas a las entrevistas aplicadas por los docentes y los estudiantes en sus grupos, las cuales se incorporaron en la base de datos en spss y que se analizaron. Estos son los aportes cualitativos que cuantificamos y tabulamos, pero que permiten ilustrar nuestros análisis. 
- A ser secuestrados: "me secuestró la expareja de mi tía". "Una vez me intentaron llevar y ahora me da mucho miedo salir". "Unos extraños en la calle me tomaron foto".

- A ser violadas: "alguien la debe acompañar a mi prima que violaron". "Me da miedo que me violen". "Ya no me dejan salir sola".

- O simplemente, como dice uno de los estudiantes: "he pasado por cosas malas que luego me afectan".

Otros hechos que ocasionan intranquilidad, susto y miedo en estos estudiantes de secundaria son las peleas y golpes entre vecinos, sobre todo cuando los lastiman a ellos mismos y a sus familias: "golpearon a mi familia y a mí también"; "mi familia se peleó con los vecinos y me pegaron"; "me espantó porque en ocasiones sacan armas o cuchillos"; "me asustó porque se mete mi familia"; "me lastiman"; "mi familia estaba en la pelea y podría correr peligro"; más allá de los chismes y burlas del vecindario acerca de sus propias familias, que también les afecta y de que en la noche tampoco pueden descansar bien a causa de los problemas que ocasionan aquellos que se drogan en las calles ya que "fuman (droga) y tiran lo que sobra y nos da el humo" y "porque me marea el olor y no es agradable".

$\mathrm{Al}$ miedo que genera este conjunto de situaciones sociales violentas se suman el susto, el sentimiento de la inseguridad que se deriva de los procesos de robo o despojo de sus bienes materiales, ya sea que se haya realizado con o sin violencia, como los robos en su casa, de sus terrenos. "Le restaron seis metros a la propiedad de mí familia”; del automóvil e incluso "en el local de mi papá, y los asaltos: "al momento del acto nos asustamos mucho; llevó mis cosas y no quiero que me las roben"; "no quiero que me roben mis cosas que me costaron mucho esfuerzo"; "me afecta personalmente y mentalmente"; "están en la calle y da inseguridad porque pueden meterse a robar a mi casa y me siento insegura"; "me siento inseguro en la casa donde vivo"; "se pueden meter a robar a mi casa y me da mucha inseguridad."
Así, la percepción de los estudiantes es que 'la calle es más peligrosa' y les da miedo e inseguridad: "porque algunas veces de noche salgo sola"; "me da miedo salir a la calle";" si siguen pasando este tipo de atrocidades ya nadie puede salir de noche porque ya no hay seguridad".

De este modo, los estudiantes o sus familiares toman como medida de precaución que los primeros salgan muy poco o que ya no salgan a la calle: "ya no me dejan salir como antes a tardes horas"; "ya no me dejan salir sola"; "por las dudas no me dejan salir solo"; "no salgo de mi casa"; "casi no salgo."

Se comprende entonces porqué estos estudiantes y sus familias no normalizan los hechos de violencia en la colonia que repercuten directamente sobre sus vidas o sobre sus pertenencias materiales o de su familia.

Desde esta perspectiva, la 'violencia' es resultado de una relación social que se materializa en una acción que tiene efectos, en primer lugar, en el cuerpo de quien ha recibido la acción, considerando que 'el cuerpo es la mediación de las relaciones sociales' (Marín, 1995). Esas acciones son concretamente la pérdida física o social de la misma vida (asesinatos, levantones, desaparición); heridas físicas y psicológicas (violaciones, peleas y golpes, maltrato); el uso violento de su cuerpo como mercancía (secuestro; efectos del consumo colectivo de drogas).

También es considerada 'violencia' la acción surgida de la relación social entre aquel sujeto que se apropia de los objetos materiales de otro sujeto a través del robo o asalto. Finalmente, son consideradas como 'violencia' las acciones que surgen de las relaciones sociales entre aquellos que desarrollan negocios ilícitos como el narcomenudeo y/o que consumen droga en las calles de la colonia.

Es decir, no solamente los sujetos encuestados no han normalizado ciertos tipos de violencia, sino que al manifestarlo a través del observable de 'hechos de violencia' se refieren a aquellos que, desde el entramado de sus relaciones sociales, experimentan en sus cuerpos o en el despojo de sus pertenencias materiales. 


\section{En el ámbito de las familias}

Según un poco menos de la mitad de los estudiantes encuestados la manera más común y recurrente es la pelea, ya sea verbal y/o a golpes entre sus diferentes integrantes o hacia ellos. Otro de los hechos que han considerado explícitamente como violencia familiar es la ruptura de sus lazos más estrechos: la separación o divorcio entre sus padres - casi siempre precedido de golpes y peleas entre ellos- y el abandono del hogar por parte de la madre. Los estudiantes reconocen que este conjunto de hechos tienen como efecto el golpe a su autoestima, la tristeza, la depresión, de que no se hable entre los miembros de la familia, además del miedo. Sin embargo, un pequeño grupo de estos estudiantes afirma que estos hechos no les afecta o no les importa porque es asunto entre sus padres o de las personas de la familia que pelean entre sí.

Finalmente, otra serie de hechos violentos en el ámbito familiar que señala el $5 \%$ de los estudiantes, respecto de un familiar, es su asesinato o intento (heridos de bala); violación o intento de violación y el secuestro. El efecto inmediato que reconocen en ellos es el terror que se apropia de la familia. De este modo, los hechos de violencia en la familia también tienen que ver con la afectación directa sobre el cuerpo, con heridas físicas y/o psicológicas; en menor medida, con la misma vida (asesinatos) y con el uso del cuerpo como mercancía (secuestro), además de otros hechos como la ruptura del tejido familiar y el abandono del hogar. Del mismo modo que en la colonia, estos hechos violentos en las relaciones familiares afectan de manera directa a sus cuerpos.

\section{En la escuela y en el aula}

En el caso de la gran mayoría de los estudiantes, los hechos de violencia en el ámbito escolar más mencionados son los existentes entre pares: desde peleas verbales hasta peleas a golpes, el acoso y la simultaneidad entre éste y las peleas y el robo. Otros estudiantes prefieren no explicitar el tipo de problemas que padecen porque 'nos iría peor'. También consideran cómo ciertas formas en la relación social de ellos con los docentes agregan una dosis de violencia (véase tabla 2).
Tabla 2. Tipos de hechos de violencia en la escuela según los estudiantes de secundaria

\begin{tabular}{|l|l|}
\hline $\begin{array}{c}\text { Relaciones } \\
\text { sociales }\end{array}$ & \multicolumn{1}{c|}{ Tipo de violencias, según los estudiantes } \\
\hline $\begin{array}{l}\text { Entre } \\
\text { estudiantes }\end{array}$ & $\begin{array}{l}\text { Peleas y golpes, acoso escolar (bullying); peleas } \\
\text { y acoso escolar (bullying) simultáneamente; } \\
\text { robo; "muchos problemas". }\end{array}$ \\
\hline $\begin{array}{l}\text { Entre } \\
\text { estudiantes, } \\
\text { docentes y } \\
\text { prefectos }\end{array}$ & $\begin{array}{l}\text { Ante el robo, "me culpan de agarrar el dinero } \\
\text { de mis compañeros"; Ante peleas y/o acoso o } \\
\text { robo: "El maestro no hace caso"; "nos dejan } \\
\text { sin recreo"; "nos ponen reporte o citatorio", } \\
\text { "culpan a todo el grupo". }\end{array}$ \\
\hline
\end{tabular}

Fuente: elaboración propia (2016).

De los estudiantes que mencionan estos hechos de violencia en la escuela y en el aula, sólo tres de cada diez admiten que les ha afectado directamente tanto en su relación entre pares como en la relación de los docentes hacia ellos (véase tabla 3 ).

Tabla 3. Efectos de las violencias en la escuela y en el aula según los estudiantes

\begin{tabular}{|l|l|}
\hline $\begin{array}{c}\text { Relaciones } \\
\text { sociales }\end{array}$ & \multicolumn{1}{c|}{ Efecto de las violencias } \\
\hline \multirow{2}{*}{$\begin{array}{l}\text { Entre } \\
\text { estudiantes }\end{array}$} & $\begin{array}{l}\text { Lastiman su autoestima y el cuerpo: enojos, } \\
\text { discriminación, "me duelen", "nos afecta a todos } \\
\text { porque algunos se unen a los abusadores y otros } \\
\text { retiran la mirada"; "Me critican por mi aspecto } \\
\text { físico", "porque hablan mal de mí", "porque les } \\
\text { caigo mal", "te hacen sentir mal y te bajan el } \\
\text { ánimo", "me afecta psicológicamente", "cuando } \\
\text { me dicen de cosas, me hacen de menos y me } \\
\text { agreden", "porque me hacen sentir menos", } \\
\text { "que me insulten porque baja mi autoestima" } \\
\text { "porque al tratar de pararlos, recibí un golpe", } \\
\text { "Salí lastimado", "Porque uno es mi amigo o } \\
\text { amigas", "porque no hay manera de pararlos". } \\
\text { El robo: "me robaron mi dinero"; "me quedo } \\
\text { sin comer y mi mamá me regaña"; "porque no } \\
\text { tengo dinero para comer". }\end{array}$ \\
\hline $\begin{array}{l}\text { Entre } \\
\text { estudiantes y y } \\
\text { docentes }\end{array}$ & $\begin{array}{l}\text { Por efectos en el aprendizaje o por las acciones } \\
\text { que toman los maestros: "no te dejan concentrar } \\
\text { en lo que estás haciendo", "nos sacan del salón } \\
\text { y no terminamos los trabajos"; el robo: "me } \\
\text { culparon y me hicieron sentir mal". }\end{array}$ \\
\hline
\end{tabular}

Fuente: elaboración propia (2016).

Para la gran mayoría de los docentes de la secundaria, el hecho de violencia en el ámbito escolar ocurre sobre todo entre los estudiantes y lo denominan bullying, sin embargo, una parte de los 
docentes menciona también hechos de violencia en el resto del entramado escolar (véase tabla 4).

Tabla 4. Hechos de violencias en la escuela y en el aula por relación social, según los docentes

\begin{tabular}{|l|l|}
\hline $\begin{array}{c}\text { Relaciones } \\
\text { sociales }\end{array}$ & \multicolumn{1}{|c|}{ Tipo de violencias según los docentes } \\
\hline $\begin{array}{l}\text { Entre } \\
\text { estudiantes }\end{array}$ & $\begin{array}{l}\text { Peleas y golpes; agresiones verbales, ya sea como } \\
\text { peleas verbales colectivas' o de manera escrita: } \\
\text { hacia un compañero con tendencia diferente o } \\
\text { por higiene bajo la forma de insultos, ofensas, } \\
\text { de poner apodos o hacer chismes; robo de las } \\
\text { mochilas; aventar o esconder las mochilas. }\end{array}$ \\
\hline $\begin{array}{l}\text { Entre } \\
\text { estudiantes y } \\
\text { docentes }\end{array}$ & $\begin{array}{l}\text { Daño material en el mobiliario o contra perte- } \\
\text { nencias de los docentes; robo a los docentes. }\end{array}$ \\
\hline $\begin{array}{l}\text { Entre } \\
\text { docentes }\end{array}$ & $\begin{array}{l}\text { Robos y daños materiales; investigaciones al } \\
\text { respecto que nunca arrojan resultados, intimi- } \\
\text { dación laboral. }\end{array}$ \\
\hline $\begin{array}{l}\text { Directivos y } \\
\text { docentes }\end{array}$ & $\begin{array}{l}\text { Actuación arbitraria de los directivos en 'hacer } \\
\text { respetar el reglamento' sin darle el lugar al } \\
\text { profesor; intimidación laboral. }\end{array}$ \\
\hline
\end{tabular}

Fuente: elaboración propia (2016).

En las relaciones entre docentes y con la directiva, aquellos perciben como 'violencia escolar' el clima escolar de intimidación laboral causado por la aplicación arbitraria de la normatividad por encima del profesor y la desconfianza entre grupos de docentes, así como la intranquilidad que sienten en la escuela, aunado al que viven en sus colonias.

Sin embargo, los hechos que más les han afectado a los docentes han sido las peleas con agresión física entre estudiantes, los hechos violentos de éstos hacia ellos y los robos.

Los principales efectos de este conjunto de violencias escolares en el proceso educativo, según los docentes, son la inhibición del proceso de enseñanza-aprendizaje sobre todo por el tiempo que deben emplear para enfrentarlos; el deterioro del ambiente de convivencia que a su vez provoca el ausentismo de los estudiantes.

Una primera reflexión en torno a este conjunto de hechos de violencia escolar se expresa como efecto de una acción de fuerza que proviene de las relaciones sociales entre quienes toman la iniciativa sobre otros sujetos que participan de esa relación, que las reciben en sus cuerpos o en sus pertenencias materiales, reafirmando con Marín (1995), que el cuerpo es una mediación de relaciones sociales.

Aun así, también para los estudiantes, en la escuela y en sus aulas, la intensidad de las acciones violentas que se producen en las relaciones sociales que conforman su entramado tienen como resultante social un costo humano menor que el que se vive en la colonia o en algunas familias sobre los cuerpos de quienes las reciben o en sus propios objetos materiales.

\section{Características del entramado socioescolar estudiado}

Los estudiantes y docentes en la escuela conforman un entramado específico de relaciones sociales, son transformados por éste y a la vez, lo transforman (Piaget, 1978; Elias, 1989) ya que "[...] un cambio [...] de las estructuras de la personalidad [...] puede considerarse, con razón, como un aspecto específico del devenir de las estructuras sociales" (Elias, 1989, p. 16), considerando que "Ambos conceptos (individuo y sociedad) tienen el carácter de procesos, como algo mutable, como algo que está en flujo continuo" (Elias, 1990, p. 16).

Vistas en su conjunto, en las representaciones sobre los hechos de violencia se destacan como rasgos la simultaneidad de diversos ámbitos de acción y de los niveles de intensidad de situaciones de violencia en los ámbitos de la comunidad, la familia, la escuela y el aula generados por el traslape de la violencia como producción histórica de un orden normativo y de aquella que se ejerce de manera reiterada e intencional para ocasionar daño, siendo que ambas perspectivas acerca de la violencia están presentes en el entramado estudiado y no se autoexcluyen, privilegiando sobre todo a este último enfoque, desde donde tradicionalmente se aborda gran parte de las investigaciones sobre violencia escolar (Olweus, 1998; Furlán, 2010, 2012). 
Los hechos de violencia que se registran en la escuela reproducen, de alguna manera, los que se generan en la sociedad más amplia. La experiencia de la simultaneidad en las violencias en diferentes ámbitos da lugar a formas de convivencia marcadas por la desconfianza, las luchas por el poder, las ofensas y la exclusión; en parte, por la ruptura de estas relaciones como su efecto principal. A nivel más profundo del entramado identificamos que las experiencias de estudiantes y docentes en torno a la violencia en la escuela y en los otros ámbitos sociales se intensifican porque se asocian con situaciones de impunidad o la falta de eficacia de los responsables de atender los hechos de violencia, social y escolar de manera más específica. Es el caso de los hechos de robo afuera y adentro de la escuela, que quedan impunes, por lo que a la experiencia de despojo se suma la de indefensión e indiferencia de los demás.

En la escuela se han sobrepuesto nuevas tensiones procedentes de acciones que son parte del proceso de la realidad de violencia o de guerra mexicana asesinatos, desparecidos, secuestrados, entre otrosy por "[...] la inseguridad permanente, de la falta de garantías y de la posibilidad latente de que las pasiones y pulsiones desborden los límites de la razón y la justicia" (Mondragón, 2014, p. 22). Esto amplía los factores que explican la creciente desigualdad social aunada a la crisis económica, el aumento del desempleo y de la economía informal, el avance del negocio de la droga y de las demás industrias delincuenciales relacionadas a ésta, con la consecuente ruptura de las redes de solidaridad y de cooperación, bajo nuevas formas que desafían a la investigación socioeducativa, ya que no hay algún ámbito social y educativo que garantice ser un espacio de paz, de seguridad, de certeza de protección de la vida.

\section{Efectos psicosociales y educativos que genera el entramado social y escolar}

La relación y simultaneidad de los diferentes hechos de violencia es lo que impacta más fuertemente la compleja experiencia de la misma que tienen docentes y estudiantes. Esta se manifiesta de múltiples maneras en relaciones de poder arbitrarias acompañados de desvalorización o situaciones de sometimiento de la persona, en los sentimientos de miedo, inseguridad, incertidumbre, aislamiento, desconfianza, impotencia ("ihagan algo, por favor!" escribía uno de los estudiantes) e incluso un sentimiento de claudicación ante el clima de hostilidad del grupo en la red de relaciones sociales del entramado que conforman.

Este traslape de violencias en diversos ámbitos produce transformaciones que sufren los estudiantes y docentes, que identifican a la escuela como el ámbito en el que ocurren más hechos de violencia. Algunos docentes expresan que "la violencia se incrementa entre todos los miembros de la institución", lo que nos habla del deterioro del clima escolar que se refiere a los parámetros de la conducta que son aceptables en todos los actores. Se puede decir que ante las transformaciones que sufren los sujetos al participar en la red de relaciones que forman parte de un entramado de guerra, la escuela no está reconociendo la necesidad de hacerse cargo de la mejora del clima escolar, de implementar mecanismos para ponerse de acuerdo en lo que es aceptable como conducta en la escuela e impulsar relaciones de respeto, cooperación y seguridad física y emocional para cada uno de los miembros de la comunidad escolar.

Tanto docentes como estudiantes nos mostraron el significado que le dan a sus vivencias en torno a las violencias en la comunidad, la familia, la escuela y el aula, es decir sus experiencias y la manera que afectan sus roles como estudiantes y como personas. A los sentimientos de tristeza y miedo por las constantes peleas entre los miembros de la familia, en algunos casos maltrato a ellos mismos, la experiencia de desgarramiento del tejido familiar se agregan los efectos de las violencias en el aula y en la escuela que, según sus palabras, dañan su autoestima y bajan el ánimo, situaciones que se acompañan de impotencia ante la arbitrariedad en la aplicación de las normas o la indiferencia ante lo que les ocurre, o la injusticia.

Es especialmente delicado lo que ocurre con los estudiantes por el proceso de adolescencia que atraviesan en el que construyen su identidad y el 
sentido del nosotros, en buena medida a partir de lo que reflejan los pares de sí mismos, y en el que desarrollan nuevas formas de socialización. Vale la pena aclarar que estamos aún ante los resultados preliminares de la investigación-intervención

\section{Aun así, se pudo intervenir...}

El proceso de intervención estuvo estrechamente vinculado al proceso de indagación, del cual se desprendieron los ejes de trabajo pedagógico. Estos se construyeron articulando temas, experiencias y situaciones sobre la convivencia con una estrategia formativa dirigida a involucrar en un proceso de concienciación y de organización para realizar acciones de cooperación a favor del aprendizaje y la convivencia a los miembros del entramado escolar: docentes, alumnos a través de sus docentes y a otros profesores con funciones de coordinadores, encargados de la disciplina, trabajo social, entre otros.

Estos ejes fueron:

1. El conocimiento de las violencias que se viven y se gestan en la escuela, el aula, la familia y la comunidad. La estrategia formativa fue sentar las bases de una comunidad de aprendizaje en la que se trabajaron los intereses de los docentes, la revisión colectiva de conceptos e investigaciones educativas sobre violencia, convivencia, clima escolar, la investigación con sus alumnos, la aplicación de cuestionarios para docentes y para alumnos, la reflexión sobre los hallazgos de la investigación y la de su experiencia como docente.

2. La reflexión sobre las relaciones entre las situaciones de violencia social con las violencias de dentro de la escuela, la reflexión sobre sus efectos en docentes y alumnos y en el clima escolar, las alternativas posibles ante la situación de violencia que enfrentan como comunidad escolar. La estrategia formativa fue la puesta en común de los resultados de los cuestionarios de docentes y de alumnos, a la luz de algunas lecturas revisadas y la construcción de propuestas para hacer frente a las situaciones de violencia que afectan el aprendizaje de alumnos y la vida cotidiana de éstos y de los docentes.

3. El diseño del programa de acompañamiento entre pares, los ajustes por docente al mismo, la metodología de investigación para documentar el proceso. La estrategia formativa fue la puesta en común de las experiencias previas, la revisión de las características de un programa orientado a las relaciones de cooperación, la construcción colectiva del programa general, con sus formas de documentación y evaluación.

4. Seguimiento, evaluación y análisis del programa entre pares $u$ otra alternativa educativa aplicada. La estrategia formativa consistió en la puesta en común de la documentación del programa aplicado, la reflexión sobre la experiencia a la luz de los conceptos revisados y de los cambios buscados, la identificación y atención a las situaciones emergentes.

Los dos primeros ejes corresponden a un proceso de concienciación en los docentes sobre las violencias en los diferentes ámbitos. Para Galtung (1998), quien habla de la violencia en el contexto del conflicto, contar con una imagen consciente y cabal del mismo, en sus aspectos profundos, sus condicionantes históricas, es una conditio sine qua non para la transformación del mismo. El proceso de concientización evita la cosificación de los actores favoreciendo que se asuman como protagonistas del proceso, capaces de dirigir la transformación del conflicto, incluyendo la propia (Galtung, 2003c).

Los ejes 3 y 4 corresponden en la visión de Galtung al momento de organización y movilización social, a la construcción colectiva de un proyecto, que parte de conocer y asumir la propia realidad (ser) para proyectarse a una realidad deseada (debe ser), estableciendo un conjunto de estrategias para lograrlo. 
A continuación, se presentan algunas de las expresiones de los docentes, que se registraron en las relatorías de las sesiones a lo largo del proceso de intervención y en los relatos de profesores que van mostrando algunos cambios en las representaciones sobre el tema de violencia y reflexiones sobre las estrategias aplicadas para hacer frente a los problemas de rezago escolar y de convivencia en la escuela. Por tratarse de varias voces, cada una se identifica con un código conformado por datos como sexo de los docentes ( $\mathrm{H}$ por hombre, $\mathrm{M}$ por mujer) un número asignado a cada docente y fecha de la sesión.

\section{Expresiones del proceso de concientización}

\section{Intereses:}

Me interesa conocer cómo influye la violencia en el desarrollo de los niños, es decir por qué no aprenden. La violencia qué tanto tiene que ver. (M.1.19-04-2016).

Me interesa el porqué de los cambios en las actitudes y comportamientos de los alumnos. (H.2.19-04-2016).

Quiero conocer que tanto yo ejerzo esa violencia con ellos. (M.3.19-04-2016).

\section{Reflexiones a partir de la experiencia y las lecturas:}

Hay descomposición en cuanto a la estructura social y económica, las reglas se usan a nuestra conveniencia y eso sucede dentro de la escuela. Las reglas se cumplen para unos y para otros no, eso confunde a los alumnos. (H.4.29-04-2016).

En las violencias que existen se van generando situaciones en las que todos estamos incluidos, aun cuando tratamos de evitarla. (H.5.29-04-2016).

Tenemos un desconocimiento de las causas de la violencia y los contextos en donde se generan, es decir, ignorancia. (H.6.29-04-2016).

Falta de comunicación entre las generaciones y la que existe entre los padres y los maestros. (M.2.29-04-2016).

La escuela actúa como si la violencia no estuviera presente. (M.7.29-04-2016).
El sometido y el que somete tienen problemas con el poder (tanto entre pares como con docentes, así mismo docente-docente). (H.8.17-05-2016).

Los alumnos no se sienten identificados con la escuela. (M.1.17-05-2016).

La escuela es para los alumnos un espacio para socializar, no para aprender y eso dificulta el proceso del aprendizaje. (H.5.17-04-2016).

La escuela le proporciona poca escucha al alumno. Tampoco los docentes tienen sentido de pertenencia. Estamos sin herramientas ante la violencia de los alumnos. (M.9.17-05-2016).

Se ejerce la violencia desde las normas de administración, desde los planes de estudio. La violencia viene desde la cúpula hacia abajo y los que pagan son los alumnos. Entonces, ¿cómo tener una convivencia pacífica dentro de la escuela? Esa sería la pregunta. (H.10.17-05-2016).

Existe también la violencia y la mala convivencia entre los docentes y tenemos que partir de ahí para poder generar convivencia entre los alumnos para poder generar un cambio. (M.11.17-05-2016).

Las expresiones vertidas por los docentes al reflexionar sobre la realidad de las violencias en ámbitos escolares y sociales representan, de acuerdo con Galtung (2003), un ejercicio de honestidad ya que se está tocando algo elemental, el texto profundo que es lo que conduce a las partes a actuar a veces sin que ellas mismas estén completamente conscientes de lo que hacen, porque ha sido suprimido, porque se ha convertido en un hábito, o simplemente porque se ve tan obvio como una expresión de lo que es normal y natural que se mantiene sin verbalizar. Este proceso dio lugar a la necesidad de actuar en torno a la problemática detectada, sin dejar de lado la necesidad de construir herramientas para la comprensión e intervención por parte de los docentes.

\section{Expresiones en el proceso de organización y movilización}

\section{Manos a la obra, acciones de cooperación:}

En los objetivos es importante propiciar que el alumno identifique qué es la violencia y por qué la asumen como algo 'normal'. (M.3.24-05-2017). 
El acompañamiento o tutoría entre pares puede ayudar a atender situaciones de violencia y actitudes para aprender y desarrollar el trabajo escolar. (H.12.24-05-2016).

Para organizar el acompañamiento entre pares debe existir empatía entre el tutor y al acompañante. (M.7.24-05-2016).

Organicé a todo el grupo en binas, los alumnos seleccionados como tutores tienen capacidad de apoyar muy bien a sus compañeros, más allá de las calificaciones que tienen. Los tutorados eligieron a sus tutores. (M.9.24-05-2016).

\section{Compartiendo reflexiones sobre el proceso:}

Existe apatía entre el tutor y el acompañante, el tutorado no se presta para escuchar las sugerencias. Es poco el avance, pero se está tratando de llevar el acompañamiento. (H.10.24-05-2016).

En mi caso, el tutor ha avanzado con su acompañante. (M.13.31-05-2016).

Aun cuando es una fase exploratoria debe existir apoyo también por parte del docente para que el logro del acompañamiento sea positivo. (H.5.31-05-2016).

\section{Valorando los resultados:}

Existen muy buenas experiencias en cuanto al trabajo de tutoría entre pares, sin embargo, es necesario el apoyo de todo el colectivo. (H.8. 21-06-2016).

La sorpresa que tuve fue que $\mathrm{K}$ misma me buscó para pedirme que si le podía recibir sus trabajos y tareas atrasadas, cuando era yo que tenía que estarle pidiendo que trabajara en clase. (M.14.21-06-2016).

Una de mis alumnas que fue tutora me comentó que además de que ayudó a un compañero se ayuda también a sí misma, el aprendizaje es mutuo. (M.15.21-06-2016).

\section{En voz de los estudiantes:}

Ser tutora es algo nuevo para mí, ya que ayudo a un compañero que le cuesta una materia. Me gusta porque me pone atención, si él tiene buen desempeño, me siento orgullosa de él. (M.16.28-06-2016).

Cuando empecé con mi tutorada no era buena, se iba con sus amigos a jugar, no tenía interés en hacer nada, faltaba; no se llevaba bien con sus compañeros. Tuve que buscar la manera de actuar con ella. Con el tiempo empezamos a llevarnos bien, ahora pone más interés en las cosas. (M.17.28-06-2016).

El acompañamiento entre pares no sólo mejoró las actitudes para aprender, sino también la autoestima y sus competencias sociales; pero no fue fácil, tuve que inventar. (M.18.28-06-2016).

En el proceso de organización y movilización que se concretó en el ámbito de la secundaria en un proceso de intervención educativa orientada a el acompañamiento entre pares como un modo de favorecer la cooperación para aprender y convivir y fortalecer el tejido social, identificamos como procedimiento central, la comunicación entre todos los actores en un diálogo en el que se pueden identificar características planteadas por Galtung (2006): la empatía, creatividad y no violencia como estrategias para la construcción de paz como el peace making que opera sobre las actitudes y el peace keaping que opera sobre los comportamientos. En ese esfuerzo se movilizaron tanto la concientización como la intervención.

\section{El esbozo de una propuesta a estudiar}

Ante el conjunto de violencias que sacude al entramado de la escuela secundaria estudiada, afectándola en el desarrollo de los procesos de enseñanza y aprendizaje, en el deterioro del ambiente de convivencia y en el rezago educativo y deserción, e integrando este estudio a la reflexión acumulada a lo largo de estos años de investigación sobre esta temática, identificamos la necesidad de que, con la participación de todos los actores se impulsen escuelas basadas en 'redes territoriales de cooperación social'. Este sería un modo de enfrentar hechos estructurales y coyunturales que se sobreponen a la escuela y los que ésta misma contribuye a generar, que tienen como efectos la producción de sentimientos de impotencia, el no saber qué hacer que experimentan los diversos miembros del entramado 
en sus relaciones sociales. Es decir, a las experiencias de convivencia basadas en relaciones sociales de violencia, desconfianza, de miedo e, incluso, de terror, se presenta como desafío epistémico la creación de condiciones de experiencias de cooperación que rompan el cerco en que se han constituido la familia, la escuela y la ruptura del tejido social en las colonias.

\section{A modo de conclusión}

El Diplomado de Violencia escolar y pedagogía de la cooperación para la ciudadanía dirigido a docentes de una escuela secundaria asentada en una de las áreas más inseguras de Morelos, México, llevada a cabo en 2016 a través de una investigación acerca de las diversas formas de violencias sociales y escolares en los diferentes ámbitos de su vida cotidiana y en la de sus estudiantes así como en un proceso de intervención de acompañamiento entre pares, evidenció la complejidad y simultaneidad de antiguas y nuevas formas de relacionamiento violento al interior del entramado socioescolar así como en la descomposición del mismo con efectos profundos en la autoestima.

Estos efectos, pueden evidenciarse en el proceso de enseñanza-aprendizaje, en la inseguridad por la misma vida y de los propios. Sin embargo, también se señala cómo se constituyen en espacios posibles para acciones de cooperación al interior y al exterior del recinto escolar - aún no todas exploradas, por supuesto - pero marcando un desafío para las investigaciones en el terreno de la violencia escolar y mostrando la fertilidad que éstas tienen cuando se amplía su estudio al entramado de relaciones sociales de los sujetos de estudio.

\section{Referencias}

Cervantes, L., Poujol, G., y Escalante, F. (2013). De la desigualdad a la exclusión cultural en la escuela secundaria. Estudio en dos casos. Artículo en memoria electrónica del XII congreso nacional de investigación educativa. Guanajuato, México. Recuperado de http:// www.comie.org. $\mathrm{mx} /$ congreso/memoriaelectronica/v12/doc/0668.pdf

Colombo, G. (2011). Violencia Escolar y Convivencia Escolar: descubriendo estrategias en la vida cotidiana escolar. Revista Argentina de Sociología, 8-9 (15-16), 81-104.

Consejo Ciudadano para la Seguridad Pública y Justicia Penal. A.C. (2014). Seguridad, Justicia y Paz. La violencia en los municipios y en las entidades federativas de México. Recuperado de http://www.seguridadjusticiaypaz.org.mx/ biblioteca/prensa/finish/5-prensa/205-la-violencia-en-los-municipios-y-en-las-entidadesfederativas-de-mexico-2014/0

Di Napoli, P. (2009). Enfoques teóricos y ejes de debate para el estudio de las violencias en el ámbito escolar. $V$ Jornadas de Jóvenes Investigadores. Instituto de Investigaciones Gino Germani, Buenos Aires.

Elías, N. (1990). La sociedad de los individuos. Boloña: Il Mulino.

Elías, N. (1998). El proceso de la civilización. Investigaciones sociogenéticas y psicogenéticas. México: Fondo de Cultura Económica.

Equipo Bourbaki. (2011). El costo humano de la guerra por la construcción del monopolio del narcotráfico en México (2008-2009). Recuperado de http://webiigg.sociales.uba.ar/revistacuadernosdemarte/nro1/InformeBourbaki.pdf

Fracchia, M., Arredondo, B., y Mendoza, R. (2015). Efectos violentos visibles y no visibles del orden normativo en la relación entre pares en dos escuelas primarias. Memoria electrónica del XIII Congreso Nacional de Investigación Educativa. México: Consejo Mexicano de Investigación Educativa. Recuperado de http://www.comie. org. $\mathrm{mx} /$ congreso/memoriaelectronica/v12/ doc/0902.pdf

Fracchia, M., García, J., y Cortés, O. (2013). Las relaciones sociales implicadas en las violencias expresadas en dos escuelas de educación básica. Memoria electrónica del XII Congreso Nacional de Investigación Educativa. Universidad de Guanajuato. Recuperado de http://www.comie.org.mx/congreso/memoriaelectronica/ v12/doc/0902.pdf 
Furlan, A. (2012). Reflexiones sobre la violencia en las escuelas. México: Siglo Veintiuno Editores.

Furlán, A., y Spitzer T. C. (2013). Convivencia, disciplina y violencia en las escuelas. México: Consejo Mexicano de Investigación Educativa.

Galtung, J. (2006). La trasformazione dei conflitti con mezzi pacifici (Il Metodo TRANSCEND). Torino: United Nations Disaster Management Training Programme- Centro Studi Sereno Regis.

Galtung, J. (1998). Tras la violencia, 3R: reconstrucción, reconciliación, resolución. Afrontando los efectos visibles e invisibles de la guerra y la violencia. Bilbao: Gernika Gogoratuz.

Galtung, J. (2003c). Paz por medios pacificos. Paz y conflicto, desarrollo y civilización. Bilbao: Gernika Gogoratuz.

Jiménez, F. (2012). Conocer para comprender la violencia, origen causas y realidad. Convergencia, Revista de Ciencias sociales, (58), 13-52.

Kaplan, C. (2009). Violencia escolar bajo sospecha. Buenos Aires: Miño y Dávila.

Krauskopf, D. (2006). Estado del arte de los programas de prevención de la violencia en ámbitos escolares. Washington D.C: Pan American Health Organization y Cooperación Técnica Alemana-gtz.
Marín, J. C. (1995). Conversaciones sobre el poder. Buenos Aires: Universidad de Buenos Aires.

Mondragón, A. (2014). ¿Estado de derecho o estado de Guerra? En G. Contreras, J. Flores, A. Mondragón y I. Saavedra (eds.), No nos alcanzan las palabras. Sociedad, estado y violencia en México. México: UAm-Itaca.

Onwuegbuzie A. J., \& Leech, N. L. (2006). Linking Research Questions to Mixed Methods Data Analysis Procedures. Qual Report; 11(3), 474-498. Recuperado de http://www. nova. edu/ssss/QR/QR11-3/onwuegbuzie.pdf

Piaget, J. (1978). Introducción a la epistemología genética: el pensamiento sociológico. Buenos Aires: Paidós.

Poujol, G. (2016). Puntos de articulación entre violencia social, exclusión y violencia escolar. RevistaLatinoamericanadeEstudiosEducativos, XLVI (2), 123-144. Recuperado de http:// www.redalyc.org/pdf/270/27046182006.pdf

Poujol, G., y Sánchez, S. (2015). Ambiente sociomoral como entramado: individuo, historia, sociedad y cultura. Memoria electrónica del XIII Congreso Nacional de Investigación Educativa. Guanajuato, México. 